\title{
Correlative Microscopy Using TEM and SIMS: Parallel Ion Electron Spectrometry (PIES) for High-Resolution, High-Sensitivity Elemental Mapping for Applications in Materials Science and Biology
}

\author{
Santhana Eswara Moorthy, David Dowsett and Tom Wirtz
}

Department "Science and Analysis of Materials" (SAM), Centre de Recherche Public - Gabriel Lippmann, 41 rue du Brill, L-4422 Belvaux, Luxembourg

The capability to simultaneously obtain high spatial resolution and high chemical sensitivity is of paramount importance to gain deeper understanding in physical and biological sciences. The Transmission Electron Microscopy (TEM) offers superior spatial resolution, but, the traditional analytical capabilities associated with electron microscopy such as the Energy Dispersive Spectroscopy (EDS) or Electron Energy-Loss Spectroscopy (EELS) are unfortunately inadequate for characterizing samples containing trace elements (at best 0.1 at. \%) or for mapping isotopic distributions [1,2]. On the other hand, Secondary Ion Mass Spectrometry (SIMS) provides extraordinary chemical sensitivity (down to ppm or even ppb) and high dynamic range, but, offers poor lateral resolution [3]. An ex-situ combination of TEM and SIMS in an attempt to overcome the limitations of the techniques taken individually is prone to sample modifications and other artefacts [4]. To overcome the intrinsic instrumental limitations, we have made an in-situ combination to complement the high-sensitivity of SIMS with the exceptional spatial resolution offered by TEM, by developing the correlative TEM-SIMS technique.

To determine the feasibility and to demonstrate the applications of the TEM-SIMS technique, we have developed a prototype instrument for TEM-SIMS based correlative microscopy (Figure 1). The polepieces of a Tecnai F20 were specially modified to accommodate the SIMS technique. A commercial FEI Magnum $\mathrm{Ga}^{+}$FIB was attached to the TEM column to act as the primary ion column. The secondary ion extraction optics (extraction efficiency 90\%) and a compact high-performance mass spectrometer were designed and developed in-house and are being continuously improved for optimal performance. A special sample holder which can be biased to high-voltages $( \pm 4.5 \mathrm{kV})$ was also developed in-house to enhance the collection efficiency of the secondary-ion extraction optics.

To enhance the low intrinsic yield of secondary ions for non-reactive primary ion beams such as $\mathrm{Ga}^{+}$for the TEM-SIMS we use reactive gas flooding [5]. Specifically, the enhancement of negative secondary ion yields due to Cs flooding and of positive secondary ion yields with $\mathrm{O}_{2}$ flooding were found to be up to four orders-of-magnitude. This enhancement of secondary ion yields leads to detection limits varying from $10^{-3}$ to $10^{-6}$ for a lateral resolution between $10 \mathrm{~nm}$ and $100 \mathrm{~nm}$ respectively (Figure 2).

In this presentation, the strategies employed to overcome the technical challenges of the TEM-SIMS combination of techniques will be discussed and the new possibilities enabled by this correlative microscopy method will be highlighted with a focus on applications in materials science and biology. Special emphasis will also be placed on TEM-SIMS characterization of samples containing light elements (low Z), which are particularly challenging with traditional analytical methods like EDS or EELS. 


\section{References:}

[1] L. Reimer, H. Kohl, Transmission Electron Microscopy: physics of image formation (Springer, New York, 2008).

[2] R. F. Egerton, Electron Energy-Loss Spectroscopy in the Electron Microscope (3rd edition), (Springer, New York, 2011).

[3] D. S. McPhail, J. Mater. Sci. 41, (2006) 873.

[4] K. Q. Ngo et al, Surf. Sci. 606, (2012) 1244.

[5] P. Philipp et al, Int. J. Mass. Spectrom. 253 (2006) 71

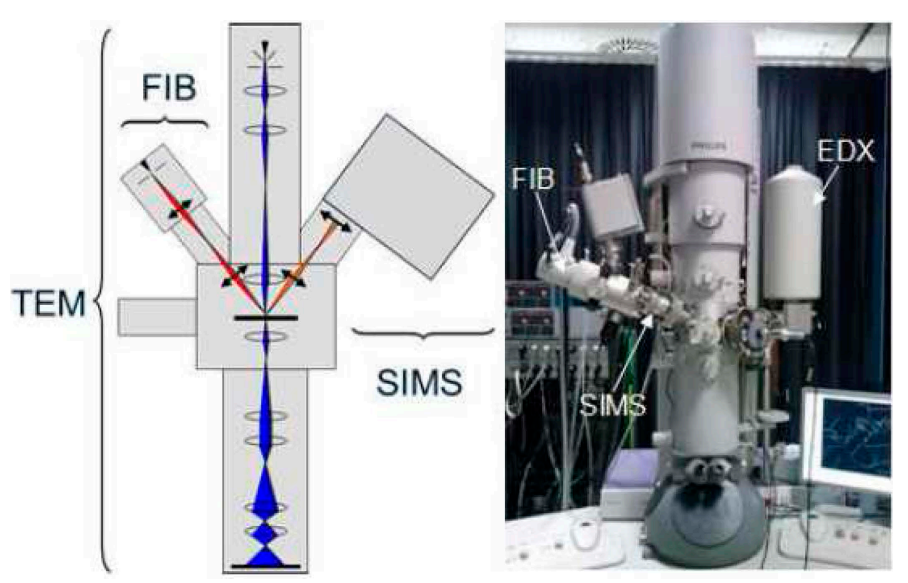

Figure 1. Schematic of the TEM-SIMS setup (left) and the photo of the TEM-SIMS prototype instrument (right).

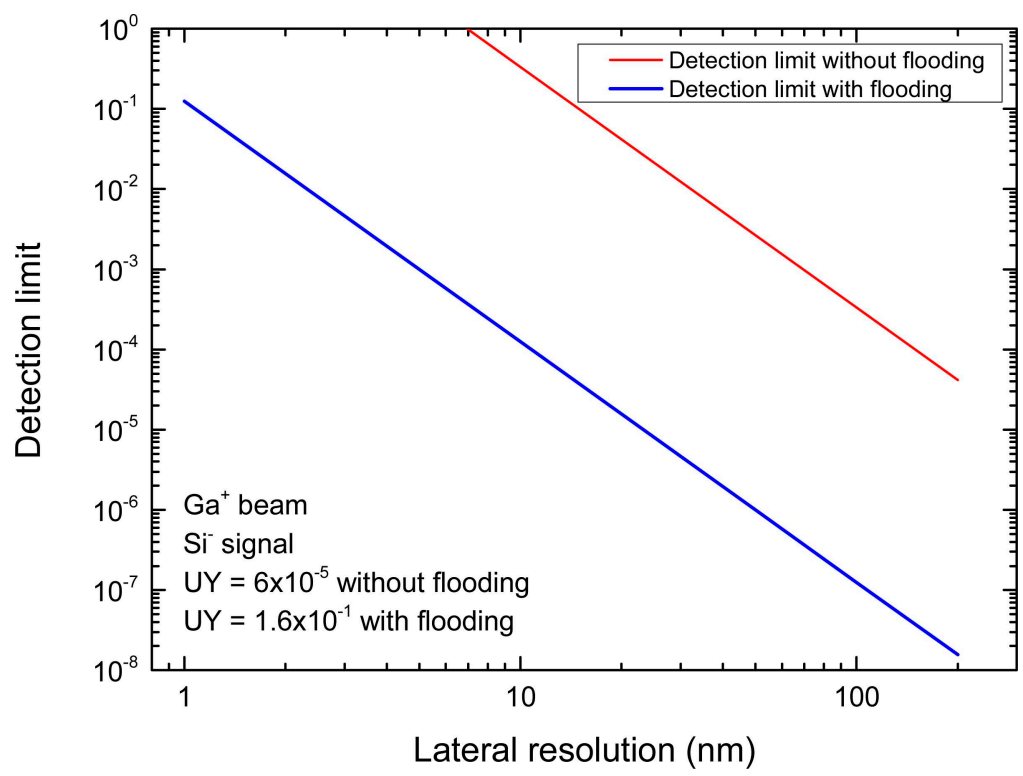

Figure 2. Detection limit using a $\mathrm{Ga}^{+} \mathrm{FIB}$ with and without $\mathrm{Cs}^{0}$ flooding vs. minimum feature size: example for the detection of $\mathrm{Si}^{-}$. 